\title{
Novel use of the endobronchial ultrasound through esophagus (EUS-B): Diagnosis of thyroid lesions
}

\author{
Mario Tamburrini ${ }^{1}$, Parikshit Thakare ${ }^{2}$, Umberto Zuccon ${ }^{1}$ \\ ${ }^{1}$ Department of Internal and Specialist Medicine, Unit of Pneumology, Friuli Venezia Giulia Health Authority \\ Hospital, Pordenone, Italy; ${ }^{2}$ Department of Pulmonary Medicine, TNMC \& BYL Nair Hospital, Mumbai, India
}

\begin{abstract}
There is paucity in literature on the use of endobronchial ultrasound through esophagus (EUS-B) for the diagnosing thyroid gland lesions. We report the first case of colloid goiter diagnosed using EUS-B-FNA technique. A 77-year-old man presented with ophthalmic symptoms and an incidental finding of lung nodule on chest $\mathrm{x}$-ray. The computed tomography of thorax revealed a left upper lobe nodule and an oval shaped left paratracheal lesion near left pole of thyroid gland. EUS-B-FNAC was performed which lead to the diagnosis of colloid goiter.
\end{abstract}

\section{Introduction}

Endobronchial ultrasound (EBUS) is very useful, accurate and minimally invasive tool for hilar and mediastinal lymph nodes structure assessment using conscious sedation. It has been increas-

Correspondence: Dr. Mario Tamburrini, Azienda Sanitaria Friuli Occidentale, S.C. Penumologia, via Montereale 24, 33170 Pordenone, Italy.

E-mail: mario.tamburrini@aas5.sanita.fvg.it

Contributions: All the authors made a substantive intellectual contribution, read and approved the final version of the manuscript and agreed to be accountable for all aspects of the work.

Conflict of interest: The authors declare that they have no competing interests, and all authors confirm accuracy.

Informed consent: Written informed consent was obtained from the patient.

Key words: Lymphadenopathy; endobronchial ultrasound through esophagus (EUS-B); thoracocentesis.

Received for publication: 17 March 2020.

Accepted for publication: 10 April 2020.

${ }^{\circ}$ Copyright: the Author(s), 2020

Licensee PAGEPress, Italy

Monaldi Archives for Chest Disease 2020; 90:1275

doi: 10.4081/monaldi.2020.1275

This article is distributed under the terms of the Creative Commons Attribution Noncommercial License (by-nc 4.0) which permits any noncommercial use, distribution, and reproduction in any medium, provided the original author(s) and source are credited. ingly used to biopsy other hilar and midline accessible structures $[1,2]$. When an EBUS scope is passed through the esophagus to assess the mediastinal structures, it is termed an Endobronchial Ultrasound thorough esophagus (EUS-B). It is being frequently used to sample the mediastinal structures through the esophagus [3]. It provides nearly complete access to all relevant lymph nodes for staging lung cancer; permits the diagnosis of para-esophageal mediastinal and lung lesions which cannot be accessed through the tracheo-bronchial tree. It also permits access to the lower mediastinal lymph node stations (e.g., stations 8 and 9) and subdiaphragmatic lymph nodes. A few case reports cite the use of EBUS to sample paratracheal lesions which led to the diagnosis of thyroid disease. However, there is paucity in literature on the use of EUS-B for the diagnosing thyroid lesions. We report the first case of a colloid goiter diagnosed using the EUS-B.

\section{Methods}

A written informed valid consent was taken from the patient. BUS was performed using a convex probe EBUS Olympus-BFUC180F EBUS scope and the EU-ME2 ultrasound (Olympus). The procedure was performed by two pulmonologists, a nursing staff and was done under conscious sedation with a mouth guard using intravenous midazolam and fentanyl as advised by the BTS guidelines for bronchoscopy. Local anesthesia was achieved by spraying $10 \%$ lignocaine in the posterior pharynx prior to the procedure. The bronchoscope was passed by one bronchoscopist via the mouth guard. The routine bronchoscopy evaluation was done to visualize vocal cords, trachea and carina following which an EBUS study was attempted. In view of difficulty in recognizing the left paratracheal lesion on EBUS study due to angulation and restricted movement of the scope; negotiating and locating the lesion with EBUS scope and biopsy needle in situ was impossible. An EUS-B was carried out with a better window for the biopsy and direct approach of the lesion. The sampling procedure followed the usual standard using 22 G Cook's biopsy needle and 25 G cook's needle (EUS-B-FNA). Enlarged left paratracheal lesion were identified using digitally captured ultrasound images. The 22-gauge Olympus needle was used to puncture the lymph nodes and after each pass, slides were prepared and stained with toluidine blue to identify lymphocytes, granulomas and atypical cells. Up to three passes were made for the left paratracheal lesion and rapid on-site evaluation (ROSE) was done, which was not suggestive of any presence of bronchial cells, malignant cells or lymph node tissue. The slides were then air dried and sent for cytopathological analysis and acid-fast bacilli stain. The bronchial washing and bronchial aspirate were negative for mycobacterial and viral 
culture and malignant cells. The final pathological report was then obtained.

\section{Case report}

A 77-year-old man was hospitalized for left eye vision impairment. He had comorbidities in form of diabetes mellitus and systemic hypertension for 10 years optimized on medical line of management. He had past history of tonsillectomy done in childhood. He worked as a merchant. He was a current smoker with significant smoking index of 20 pack years. The general examination and systemic examination were normal, except ophthalmological abnormality. The detailed eye examination revealed mild hypertensive angiopathy with visual field examination diagnosing a visual field defect on left side. The other neurological clinical assessment of the patient was normal with no sensory, motor defect. The blood examination was within normal range with serum creatinine being $1.63 \mathrm{mg} / \mathrm{dl}$. The initial computed tomography of skull (CT skull) done in emergency department was suggestive of heterogeneous hypodensity in the right occipital site likely to be vascular in origin. The chest X-ray detected a pulmonary nodule in the left upper lobe. The Carotid doppler study suggested of carotid atherosclerosis with stenosis at the carotid bifurcation $25 \%$ on the right and $30 \%$ on the left. In view of persistent visual field defect, the patient underwent a contrast enhanced computed tomography of skull, thorax and abdomen. The skull scans revealed right occipital hypodensity areas associated intense gyriform enhancement due to ischemia, which was less evident than the previous CT skull in emergency department. The computed tomography of thorax confirmed a left upper lobe nodule of size $1 \mathrm{~cm}$ (Figure 1) and left paratracheal oval shaped opacity of size $3 \mathrm{~cm}$ close to the lower pole of left thyroid gland with heterogenous enhancement post contrast (Figure 2). The computed tomography of abdomen was normal. The thyroid function tests and Anti-thyroid peroxidase (Anti-TPO) antibodies were normal and MRI findings of the skull was also suggestive of ischemic changes in right occipital areas.

In clinico-radiological correlation, the patient was kept under observation as ophthalmic symptoms remained stable and planned for endobronchial ultrasound guided fine needle aspiration (EBUS-FNA). The procedure was done as described and biopsy sample was taken through the transesophageal route (EUS-BFNA) (Video 1). Subsequently the final cytology report of the EUS-B-FNA revealed colloid goiter and was negative for malignancy (Figure 3).

The final diagnosis was colloid goiter with left visual field defect due to right occipital ischemia.

\section{Discussion}

Interventional Pulmonology has grown in recent years due to the introduction of endobronchial ultrasound (EBUS) as a staging modality for lung malignancies and as a diagnostic tool for the mediastinal involvement. More recently, the efficacy and utility of the esophageal approach using the EBUS scope (EUS-B) has been described to access nodes and lesions which are accessible through it. The transesophageal and gastric use of the EBUS scope (EUS-B) was first reported in 2007 [4]. Subsequent papers proved that the dual use of EBUS, through the tracheal-bronchial tree and the esophagus could be performed in the same setting with a high diagnostic

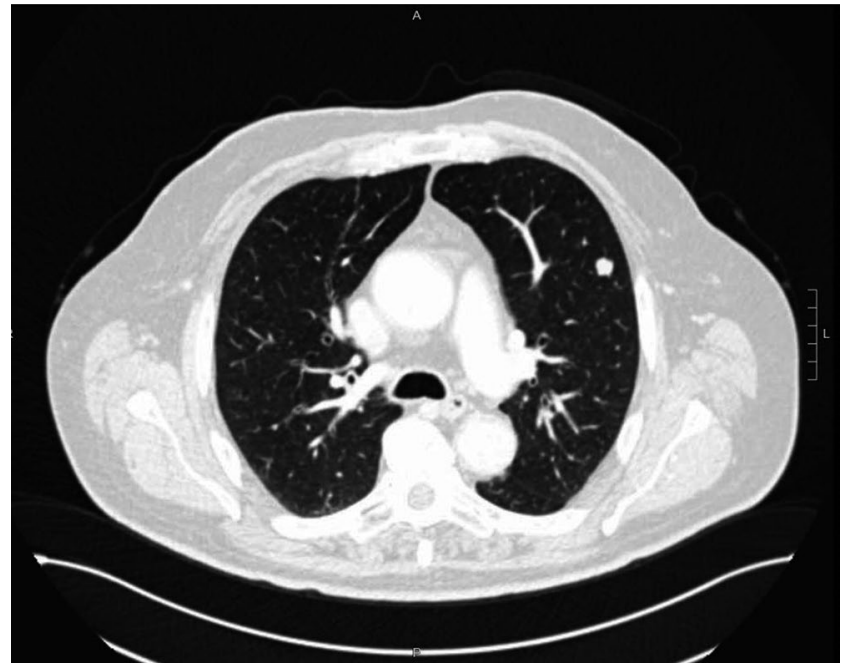

Figure 1. Computed tomography of thorax showing left lung nodule (axial view).

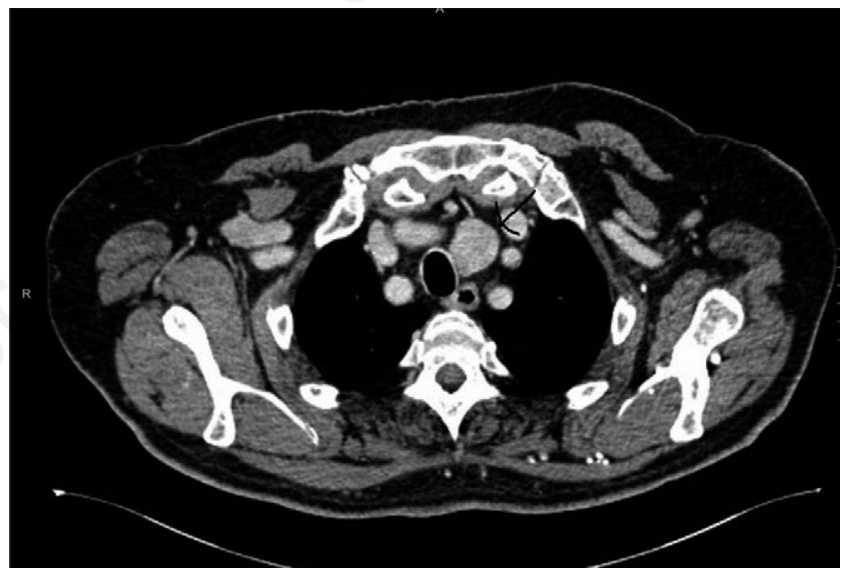

Figure 2. Contrast enhanced computed tomography thorax image showing oval opacity in left paratracheal area (axial view).

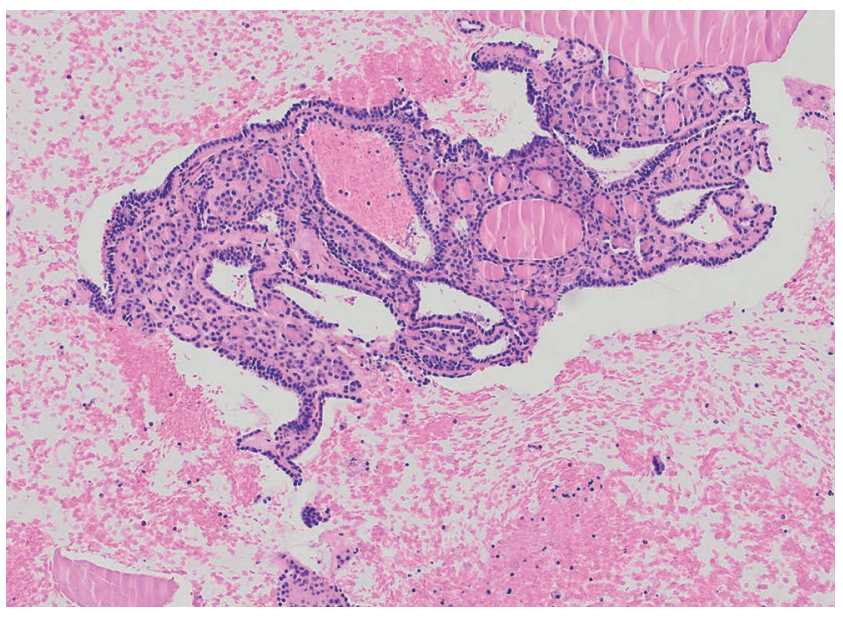

Figure 3. Histopathology image showing colloid goiter. 
yield for lung cancer staging in trained hands [5,6]. Bugalho et al. discuss the uses of EUS-B in non-malignant diseases. They discuss its role in sarcoidosis [7]. The diagnosis of pulmonary sarcoidosis in the era of EBUS/EUS-B has shifted from the erstwhile conventional flexible bronchoscopy. In recent years, the detection of non-caseating granulomas in mediastinal lymph nodes was easily performed by EBUS-FNA or EUS-FNA [8,9]. In a 2013 study, Oki et al. used EUS-B-FNA in 33 patients to diagnosis stage I/II sarcoidosis [10] and obtained a diagnostic yield of $86 \%$ without complications. EUSB-FNA diagnosed tuberculosis lymphadenitis in both children and adults when assessed with during the differential diagnosis of mediastinal or hilar lymphadenopathies $[11,12]$. In these situations, the diagnostic yield increased if samples were sent simultaneously for microbiology, cytopathology, and histopathology [13]. The EUS data from department of Gastroenterology, Topiwala National Medical College and BYL Nair Charitable Hospital, Mumbai, Maharashtra, India suggestive of EUS can be used for the diagnosis of various benign diseases [14]. Apart from this the EBUS has been also used for the aspiration of pericardial effusion [15].

The interest in the endoscopic evaluation of thyroid developed with the widespread use of EUS as it can visualize the inferior portions of the thyroid gland at around 18-20 centimeters distance from the incisors [16]. To our knowledge, only one case of thyroid cancer (Hurtle cell neoplasm) has been diagnosed by using EUS-FNA. In that case, the tumor was described as an irregular, hypoechoic, and heterogeneous mass with anechoic areas [17]. EUS-FNA and EUSB-FNA have been reported utility for diagnosing cases of metastatic (rather than primary papillary) thyroid cancer to the pancreas, lung, and lymph nodes [18-21]. A single case of parathyroid adenoma in a asymptomatic patient with primary hyperparathyroidism diagnosed with EUS-B has been reported [22].

To our knowledge this is the first case where EUS-B has been used to diagnose thyroid lesion in the form of colloid goiter

\section{Conclusions}

EUS-B can be a very important tool along with EBUS to assess and biopsy accessible mediastinal structures, hilar structures, paratracheal lesions in experienced hands with good results to avoid multiple hospital visits. This could be also of best use where EBUS is available but EUS facilities are lacking. Thus, EUS-B can be used as a novel technique to diagnose a thyroid lesion like colloid goiter.

\section{References}

1. Gomez M, Silvestri GA. Endobronchial ultrasound for the diagnosis and staging of lung cancer. Proc AmThor Soc 2009;6:180-6.

2. Du Rand IA, Barber PV, Goldring J, et al. British Thoracic Society guideline for advanced diagnostic and therapeutic flexible bronchoscopy in adults. Thorax 2011;66:iii1-21. doi: 10.1136/thoraxjnl-2011-200713.

3. Dietrich, CF Annema JT, Clementsen P, et al. Ultrasound techniques in the evaluation of the mediastinum, part I: endoscopic ultrasound (EUS), endobronchial ultrasound (EBUS) and transcutaneous mediastinal ultrasound (TMUS), introduction into ultrasound techniques. J Thorac Dis 2015;7:311-25.

4. Gupta K, Mallery S. Small-caliber endobronchial ultrasonic videoscope: successful transesophageal and transgastric FNA after failed passage of a standard ultrasonic endoscope. Gastrointest Endosc 2007;66:574-7.

5. Herth FJ, Krasnik M, Kahn N, et al. Combined endoscopicendobronchial ultrasound-guided fine-needle aspiration of mediastinal lymph nodes through a single bronchoscope in 150 patients with suspected lung cancer. Chest 2010;138:790-7.

6. Hwangbo B, Lee GK, Lee HS, et al. Transbronchial and transesophageal fine-needle aspiration using an ultrasound bronchoscope in mediastinal staging of potentially operable lung cancer. Chest 2010;138:795-802.

7. Bugalho A, de Santis M, Szlubowski A, et al. Trans-esophageal endobronchial ultrasound-guided needle aspiration (EUS-BNA): a road map for the chest physician. Pulmonology 2018; 24:32-41.

8. von Bartheld MB, Dekkers OM, Szlubowski A, et al. Endosonography vs conventional bronchoscopy for the diagnosis of sarcoidosis: the GRANULOMA randomized clinical trial. JAMA 2013;309:2457-64.

9. Gupta D, Dadhwal, DS Agarwal R, et al. Endobronchial ultrasound-guided transbronchial needle aspiration vs conventional transbronchial needle aspiration in the diagnosis of sarcoidosis. Chest 2014;146:547-56.

10. Oki M, Saka H, Kitagawa C, et al. Transesophageal bronchoscopic ultrasound-guided fine needle aspiration for diagnosis of sarcoidosis. Respiration 2013;85:137-43.

11. Dhooria S, Madan K, Pattabhiraman V, et al. A multicenter study on the utility and safety of EBUS-TBNA and EUS-BFNA in children. Pediatr Pulmonol 2016;51:1031-9.

12. Medford AR, Agrawal S. Single bronchoscope combined endoscopic-endobronchial ultrasound-guided fine-needle aspiration for tuberculous mediastinal nodes. Chest 2010;138: 1274.

13. Eom JS, Mok JH, Lee MK, et al. Efficacy of TB-PCR using EBUS-TBNA samples in patients with intrathoracic granulomatous lymphadenopathy. BMC Pulm Med 2015;1:5166.

14. Junare PR, Jain S, Rathi P, et al. Endoscopic ultrasound-guidedfine-needle aspiration/fine-needle biopsy in diagnosis of mediastinal lymphadenopathy - A boon. Lung India 2020;37:37-44.

15. Hohenforst-Schmidt W, Zarogoulidis P, Steinheimer M, et al. A new endobronchial ultrasound (EBUS) application for benign and malignant pericardial effusion (PE) aspiration: Transbronchial pericardial effusion aspiration (TPEA) with a regular EBUS transbronchial (TBNA) needle under apneic nasal jet-catheter ventilation. J Biomed 2016;1:9-25.

16. De Melo SW, Wallace MB. Normal mediastinal anatomy by EUS and EBUS. In: Gress FG, Savides TJ, Bounds BC, Deutsch JC, editors. Atlas of endoscopic ultrasonography. Chichester: Wiley-Blackwell; 2012; p. 14-17.

17. Sawhney MS, Nelson DB, Debol S. The ever-expanding spectrum of GI endoscopy: EUS-Guided FNA of thyroid cancer. Gastrointest Endosc 2007;65:319-320.

18. Alzahrani AS, AlQaraawi A, Al Sohaibani F, et al. Pancreatic metastasis arising from a BRAF (V600E)-positive papillary thyroid cancer: the role of endoscopic ultrasound-guided biopsy and response to sorafenib therapy. Thyroid 2012;22:536-41.

19. Chen L, Brainard JA. Pancreatic metastasis from papillary thyroid carcinoma diagnosed by endoscopic ultrasound-guided fine needle aspiration: a case report. Acta Cytol 2010;54:640-4

20. Chow A, Oki M, Saka H, Moritani S, Usami N. Metastatic mediastinal lymph node from an unidentified primary papillary thyroid carcinoma diagnosed by endobronchial ultrasoundguided transbronchial needle aspiration. Intern Med 2009;48:1293-6. 
21. Sakairi Y, Yasufuku K, Iyoda A, et al. A solitary metastatic lung tumor from thyroid papillary carcinoma diagnosed by endobronchial ultrasound-guided transbronchial needle aspiration (EBUS-TBNA): report of a case. Surg Today 2008; 38:46-8.
22. Ghulam Sarwar, Christopher Grainge, David Arnold. Parathyroid adenoma diagnosed with endoscopic ultrasound with bronchoscope (EUS-B) guided biopsy in asymptomatic patient with primary hyperparathyroidism. EC Pulmonol Respir Med 2017;5.4:191-5. 